10.1515/ausfm-2015-0012

\title{
Brand Identity, Adaptation, and Media Franchise Culture
}

\author{
Katherine Marazi \\ Aristotle University of Thessaloniki \\ E-mail: amarazi@enl.auth.gr
}

\begin{abstract}
In spite of the noticeable practices within the field of Adaptation, Adaptation theory seems to be lagging behind whilst perpetuating various fallacies. Geoffrey Wagner's types of Adaptation and Kamilla Elliott's proposed concepts for examining adaptations have proved useful but due to their general applicability they seem to perpetuate the fallacies existing within the field of Adaptation. This article will propose a context-specific concept pertaining to Media Franchise Culture for the purpose of examining Adaptations and re-assessing long-held debates concerning the Original, the Content/Form debate and Fidelity issues that cater to the twelve fallacies discussed by Thomas Leitch.
\end{abstract}

Keywords: brand identity, media franchise culture, adaptation, superheroes, and branded entertainment.

Brand Identity, consisting of a Core and Extended Identity in addition to Brand Equity offer a new perspective from which to view adaptations whilst taking into consideration narrative media alongside elements that have been ignored such as the Industry and consumer audiences. While Brand Identity theory is capable of tackling all twelve fallacies, focus will be placed on the issue of fidelity in juxtaposition to the new angle offered by the Core, instead of an original text, and the Extended Identity instead of content/form.

In his insightful 2003 article, Twelve Fallacies in Contemporary Adaptation Theory, Thomas M. Leitch discusses the twelve fallacies pertaining to adaptation theory and successfully highlights that, in regards to contemporary adaptation theory, the "flood of study of individual adaptations proceeds on the whole without the support of any more general theoretical account of what actually happens, or what ought to happen, when a group of filmmakers set out to adapt a literary text" $(2003,149)$. He goes on to examine fallacies pertaining to media characteristics between novels and films, their mode of representation, their 
narrative capabilities, their status as intertexts, and matters of originality. What this article will focus on are two fallacies which are interrelated and essentially occur due to the very practice of Adaptation. Both the notions of the novel being better than the film as well as the persistence that "fidelity is the most appropriate criterion to use in analyzing adaptations" cater to the degree of faithfulness an adaptation displays towards its original, meanwhile fueling debates regarding the aforementioned fallacies, whilst simultaneously acting as the point of reference when examining these fallacies (Leitch 2003, 161). The "which is better" debate of Adaptation theory as a question disregards vital aspects of the industry ${ }^{1}$ and the collaborative production process, not to mention the possible conflicting interests of licensor and licensee parties ${ }^{2}$ as well as the reception of consumer audiences. While Geoffrey Wagner may have been one of the first to identify three helpful types of adaptation, and Kamilla Elliott has successfully informed us of the proposed concepts employed to examine the form/content debate, in truth and concurrence with Leitch the notion of fidelity "is a hopelessly fallacious measure of a given adaptation's value because it is unattainable, undesirable, and theoretically possible only in a trivial sense" $(2003,161)$. Instead, Linda Hutcheon's proposition is more accurate, more inclusive of the various notions pertaining to fallacies and takes into account the element of practice.

Initially by viewing an adaptation as a formal entity, or product, one realizes that an adaptation is "an announced and extensive transposition of a particular work or works" whereby the element of "transcoding' can involve a shift in medium, or a change of frame and therefore context" (Hutcheon 2006, 7-8). The second perspective views adaptation as "a process of creation" due to the fact that the process of adaptation involves "both (re-) interpretation and then (re-) creation" $(2006,8)$. Finally, an adaptation needs to be viewed from "its process of reception" because it is "a form of intertextuality: we experience adaptations as palimpsests through our memory of other works that resonate through repetition with variation" $(2006,8)$. In an attempt to avoid repeating the fallacies Leitch refers to, this article wishes to propose a concept for the examination of adaptations within a specific context instead of offering a concept that would

1 See Johnson: “As opposed to an operation emulating vertical integration, the media franchise frequently aims for horizontal organization and cooperation" $(200947,53)$.

2 See Grainge: "Branding, [Celia Lury] suggests, emerges as an increasingly significant production strategy in a market where the basis of commercial, legal and cultural authority has changed. This not only affects the production of cultural work, witnessing the increase, for example, of titles and characters designed to be licensed across other products, but also shapes rights ownership in other areas, such as the legal protections given to star image" $(2008,11)$. 
be considered generally applicable. The context in question is that of Media Franchise Culture and the concept is that of Brand Identity theory, which can act as a model in the examination of adaptations, whilst re-evaluating long-held views, pertaining to fallacies, such as the notion of the original, the content/ form debate and the issue of fidelity. Media franchise culture ${ }^{3}$ is an area where one needs to re-evaluate what and how an I.P. is being adapted in juxtaposition with traditional notions of Adaptation theory. Cross-distribution of narratives, trans-media storytelling, media convergence, and intertextuality are not only evident but dominating practical applications in the creation of franchises. The context Adaptation theory finds itself in, therefore, is not one of simply transferring the novel to a film, nor is it about questioning whether content can be separated from form, or if the adaptation is faithful to the original. Regarding form and content, the phenomenon of media convergence distinctly displays and is predicated on the fact that content is dispersed across media platforms and trans-media storytelling complements this phenomenon by drawing attention to the fact that it is not about faithfully adapting but extending the experience of narrative by way of a multimedia construct as in the cases of Joss Whedon's Buffy the Vampire Slayer (1997-2003) and the Wachowski brothers' The Matrix (1999, 2003). Franchise storytelling culture offers an array of multitexts, multimedia, and merchandise centred on narratives, characters, or worlds,

$3 \quad$ "The potential of franchises to multiply content production across media begs us to reconsider Jenkins's notion of transmedia storytelling. Are transmedia storytelling and franchise forms synonymous? Ultimately, I argue that they are not. In Jenkins's view, transmedia storytelling provides a consistent, coordinated story world in which the consumer pieces together narratives from pieces strewn across a range of media. The story is not complete without transmedia consumption, and the proliferation of content across media is therefore carefully coordinated by producers who understand each medium and endeavor to ensure unity. Media franchises can be distinguished from this particular phenomenon for three reasons. First, there is no necessary creative coordination or singular narrative structure that unites all iterations produced by a franchise system. Second, and more pressingly, media franchises need not take narrative form. Transmedia storytelling, therefore, is certainly part of franchising, but franchising is not comprehensible as either solely narrative or transmedia phenomenon. Third, media franchises act in two discreet ways either inter-medially or intra-medially thus including the factor of transmedia but not solely characterized by it. Furthermore, the nature of the franchise is not that of a puzzle but of a shared network system that unites and even differentiates content in its ultimate goal to multiply it within a medium or across media not necessarily requesting of audiences to experience each and every mode to make sense of a narrative text mainly because media franchises also seek to explore ancillary markets such as clothes and merchandising (e.g. toys) that are not of an immediate narrative context" (Johnson 2009, 59-60). 
that are formulated based on brand marketing strategies ${ }^{4}$ aiming to offer a total entertainment experience (Grainge 2008, 54). An appropriate concept, or model, that can be employed in order to examine adaptations that occur within a Media Franchise Culture is that of Brand Identity seeing that what is adapted is ultimately either a Brand Character, or a Brand World. ${ }^{5}$

By implementing brand theory in order to establish characters as brands this article will begin by extracting the components of the brand from the narrative and medium/a so as to offer an alternate way of considering and examining current adaptations, which are more prone to the tactic of trans-media storytelling acting within a strongly emerging Media Franchise Culture. In order to achieve this, David A. Aacker's theories of brand Core/Extended Identity and Brand Equity theory will be employed in juxtaposition with superhero brands and three interindustrial media manifestations, that of film, comic book, and video game so as to display that if the superhero character is treated as a brand, then fidelity is no longer the dominant issue. Instead of being hindered by the narrative, or media specificities, adaptation theory can now enter into a constructive dialogue with narrative media while the underlying connecting factor, the respective brand of enquiry, allows one to examine elements such as what is transferred, how it is transferred and represented, by whom, and what meaning, or interpretation, can be appointed due to noticeable similarities and differences. It seems that the "which is better" debate is centred on the aspects of the Extended Identity which, as this article will argue, should not be the basis on which to make fidelity comparisons. What is more, fidelity is not dismissed as a notion but is seen in a new light as a matter of loyalty and value.

4 Cf. "At the core of the contemporary phenomenon of media branding lies the abstraction of content from the constraints of any specific analogue media format. Content has come to be conceptualized in a disembodied, almost Platonic, form: any media brand which successfully gains consumer loyalty can be translated across formats to create a raft of interrelated products, which then work in aggregate to drive further consumer awareness of the media brand" (Grainge 2008, 65-66).

5 Johnson (2009) advocates that while Hollywood is still of a Conglomerate nature, that the aspect of synergy is being replaced by a franchise media culture, one that is prone to content networks that act as contested grounds for negotiation, conflict but also creativity and production of products at an intra-industrial and interindustrial level across multiple media. According to Johnson where synergy was apt at a vertical employment of content brands media franchise culture employs content brands vertically via licensing and trademarks subjected to the notions of difference intra-industrially and deference inter-industrially. Ultimately, the character brands or worlds act as a shared product, via which licensor and licensee engage in a contested yet productive dialogue so as to achieve the multiple products either within or across media. 


\section{Brand Identity: Core and Extended}

For a brand to be strong and endure an identity needs to be forged. In the case of character-driven narratives, that identity is found to be residing predominantly within the character that can act and be perceived as a brand. This, however, does not exclude instances where the identity is forged via the cases of worldbuilding. The Lord of the Rings (Peter Jackson 2001, 2002, 2003) trilogy is a case in point where the characters but, more importantly, the world in which they reside is the brand. One of the main reasons Marvel superheroes can be seen as brands, without considering their world to be of the core identity, is due to the fact that they essentially live and act in our world, or rather a replicated version of it, in other words, a possible world.$^{6}$ The Brand Identity consists of a Core and an Extended Identity. "The core identity represents the timeless essence of the brand. The core identity, which is central to both the meaning and success of the brand, contains the associations that are most likely to remain constant as the brand travels to new markets and products" (Aacker 1996, 85-86). In the case of superheroes this cannot be the narrative, because due to adaptation and trans-media storytelling that component is constantly changing and is actually a suggestive interpretation and manifestation of more abstract concepts. Moreover, the narrative does not account for the cases of merchandise which are external components to the interior narrative chronotope. Nor can it be the medium, or media manifestations, for they are also in constant flux. The salient component, however, in all the aforementioned cases is the superhero, which can be extracted and seen on its own. When considering superheroes alongside their narratives and media manifestations, what can be extracted as belonging to the Core Identity are the notions of good versus evil and the doppelganger, or metaphorical extensions of Manichean dichotomies, exemplified via the Extended Identity. In the cases of narrative, whether that is conceived via comic books, graphic novels, animated series, television series, films, or games, these aspects are always present alongside the notion of the super-prefix in connection to the notion of hero. Thor's superiority is a given due to his divine nature, Batman's is displayed via his money and physical condition, Iron Man's is seen through his intelligence and advanced technology, the Hulk's is established through his anger and strength after the gamma ray exposure, Spiderman's is achieved biologically after the bite of the

$6 \quad$ Brian McHale discusses the notion of possible worlds in Postmodernist Fiction (1996) and how they are not constructed from scratch but are influenced by and resemble our world to varying degrees. 
radioactive spider and Wolverine's is the result of a biologic healing mutation in addition to a governmental experiment. Hence, there has to be a sense of superiority which is conceived in various ways via the Extended Identity. Secondly, the fight between good and evil is vital for the existence of any super/hero. What is intriguing at this point is that depending on the villain, who also foils and complements the superhero as an alter ego, certain Manichean dichotomies are also forged in addition to the doppelganger notion. Every superhero exemplifies duality, either openly like Iron Man, or secretly like Batman and Spiderman. By extension, each villain the superhero encounters expands the double notion to include multiple dichotomies which are also ambiguous in nature. Of course the types of dichotomies evident each time in a narrative are a result of the Extended Identity. Hence, the Extended Identity allows one to concretise, interpret and manifest abstract, yet stable, Core Identity elements.

A brand identity requires an Extended Identity in order to manifest and communicate the Core. "The extended brand identity includes elements that provide texture $^{7}$ and completeness. It fills in the picture, adding details that help portray what the brand stands for" (Aacker 1996, 87-88). The Core evidently needs to be represented, promoted, communicated and this is achieved by various elements. One factor through which this is achieved is the narrative, while another factor that provides texture and completeness to the superhero brand is the medium of manifestation. Depending on the narrative a superhero brand's story may be told in the way of a serial narrative as found in comic books, or a more holistic narrative as found in film. Moreover, the characteristics and idiosyncrasies of each medium offer differentiation to the superhero brands representation. In comic books and graphic novels the superhero brands are illustrations, in animated series they are animations, and in film they may range from flesh and blood human actors to CGI. Other elements that are affected via narrative and media are the chronotope and its representation. For example, thanks to highly realistic computer graphics, the setting found in videogames may be highly reminiscent of the setting in films whereas in comic books we have a more generalized backdrop of illustrations with specific brand indicators of chronotope. The narrator is different in each case ranging and oscillating from first to third person narration, at other times omniscient and at other times limited. The written word is highly evident in comic books, whereas in film one relies more on dialogue, mise-en-

$7 \quad$ According to Johnson the theory of textures is what Battlestar Galactica (2003 miniseries) world-builder Ronald D. Moore stated as employing when re-creating the show (2009, 193-95). 
scene and editing. In videogames, on the other hand, instructions and charts play a major role in moving the action along and informing the player of the game play. When considering merchandise such as action figures, one sees that the action figure is a replica of the superhero brand in appearance and is usually not accompanied by chronotope aspects but occasionally by other elements such as props, whether they are automobile replicas, or aspects of the superhero outfit. As for clothing and insignia, these usually represent symbols that are associated with the superhero brand and raise awareness as to the respective superhero character. What is more, the Extended Identity can be treated in such a way so as to reach wider audiences, or satisfy niche audiences. Comic books are usually targeted at younger ages and hardcore fans, blockbuster films address the masses, animated series are usually reserved for children and certain merchandise is for people of all ages as well as for those who simply want the product, or are keen on collecting said items. One can see a variety of representation and manifestation in the Extended Identity and aspects of this identity do not and are not necessarily part of the Core Identity but are rather negotiable aspects, capable of reformation and change. Having distinguished the two components of the Brand Identity, the following section will demonstrate how the Core of superhero brands is extracted from the plethora of narrative media.

\section{Superhero Brand Core Identity: the Super, the Battle, and the Doppelgänger}

Extracting the Core elements of the superhero brand may be deemed difficult in light of all the narrative media that have accumulated and ultimately play a role in how one perceives any superhero. Nonetheless, this is not an impossible feat whether a plethora of media narratives are available or not; on the contrary, perhaps having more adaptations makes this process easier due to the multiple examples for comparison and juxtaposition. Upon having established who or what the brand is, even in the case of Intellectual Properties, ${ }^{8}$ one must make the comparison and juxtapose examples so as to unveil the salient Core elements of said brand. In the case of superheroes, the Core elements seen in all mainstream

8 Cf. Grainge: "commodified texts remain discursive properties and are subject to 'multiple social authorings.' If intellectual property has a cultural life, the meaning of a brand is not simply determined by those who circulate and co-ordinate mass media representations, but is also forged in cultural instances where texts, symbols and images are used by social agents, interpreted by audiences and taken up by fan groups in potentially unforeseen ways" $(2008,12)$. 
American superheroes in any media manifestation and throughout any narrative are their superiority, the battle between good and evil, and the notion of the doppelganger. In effect, when adapting any superhero brand narrative to, and across, any capable of story-telling medium those three Core elements are vital and necessary if one wants to see, and accept the brand as that of a superhero. Seeing that the Core Identity of brands in general is abstract, its interpretation and manifestation are essentially based on ideologies pertaining to the culture, broadly speaking, and to the company or industry, specifically speaking, that produces any brand. As a result, these Core elements are malleable, which results in similar, albeit different adaptations. Hence, the Core of a brand is the stable agent that grants continuity, while the Extended Identity is negotiable, malleable and may not exhibit aspects that will ever become a part of the Core Identity, an element that also deems an adaptation as a work in its own right. Similarly, adaptation theory has been advocating that an adaptation need be faithful to the original to some degree, otherwise the new product is something completely different and in no way an adaptation of the original work. Debates regarding fidelity issues have strived to produce theories that pinpoint the essence of the original which ought to be respected and essentially adapted but such a venture can easily fall into the category of copying instead of adapting. Adaptation as a general process requires changes in order for any entity to adapt to a new context/environment. Thus, while fidelity issues are justified in seeking to find the factor that remains constant so as not to lead to the production of a completely new product, it has made the mistake of attempting to locate that stable agent within areas that are, and need to be, malleable so that the stable agent can be perpetuated across time and various boundaries. Evidently, the stable agent cannot be either the narrative or the medium for these are elements that change when adaptation occurs due to factors such as the Industry, creators' interpretation of the Intellectual Property, matters pertaining to economics as well as advances in technology. Instead, what brand theory offers as the stable agent is the Core Identity. For adaptation to occur in the case of superhero brands what needs to remain stable is the superhero brand character with all the attributes and rules that follow it. This then will come into a dialogue and negotiation with narrative media factors so as to determine how the Extended Identity will be treated and formulated. This process is also applicable to the cases of Intellectual Properties where the brand is not a character but a complete world such as The Lord of the Rings trilogy, the Harry Potter world (Chris Columbus, David Yates, Mike Newell, Alfonso Cuaron, 2001-2011), The Matrix, Lost (J. J. Abrams, Jeffrey 
Lieber, Damon Lindelof, 2004-2010), the Buffy the Vampire Slayer universe and numerous others. Here the world, its context and rules as well as the characters need to be examined via Core and Extended Identity theory which will no doubt offer an intriguing study and examination.

The common core elements make all superheroes similar in that these elements are shared by all superhero brands. This immediately points to the notion of originality which does not uphold when, in spite of the examples juxtaposed, all are considered super/heroes. This also ensures loyalty to the brand because these elements are present within all superhero narrative media to varying degrees. What distinguishes superhero brands from one another as brands, as narratives and as products of one medium and/or multiple media is the malleability and treatment of these abstract elements in the area of the Extended Identity of the brand where these elements are essentially grounded, tackled, negotiated, manifested, and given texture(s). The manifestation of the super-prefix for each superhero brand grants different powers/abilities to each superhero. Their origins are also established via the super-prefix because reason needs to be given as to how these powers were accumulated, or granted, which also leads to the donning of a hidden, or open, secret identity. Continuing this train of thought, the origin account becomes a story which positions the superhero brand in a particular world/context of a specific nature with specific rules, engaging with other characters that hinder, or move, the story along, and also establishes the villains the superhero comes across, thus manifesting the battle of good versus evil. The Manichean dichotomies are also established based on the super-prefix, the origin story given to explain and rationalize it, as well as how these powers are put to use and against whom. The medium also contributes to these malleable elements by offering ways of representation for the superhero brand, his/her world and the analogous villains.

Depending on the medium other factors are added to the Extended Identity, such as comic book formats, blockbuster formats, or gaming formats, which add to the experience of any superhero brand, offering similar, or different, experiences such as story, or game play. What is more, the medium and the assigned formats negotiate and perhaps dictate how elements such as the battle between good and evil, or various Manichean dichotomies, are to be established and represented. For example, in the blockbuster format spectacle has been, and still is, an option that is preferred and has even been employed by the gaming formats for interactive experience for game players. Comic book formats, on the other hand, rely on the treatment of the gutter in juxtaposition to the treatment 
of time and space within the panels, thus leading to alternate chronotopes that need to be considered in each medium and in juxtaposition with the analogous narrative. What can be deduced at this point is that no matter what the treatment of the malleable Extended Identity has to offer the Core Identity, what is certain is that not all the aforementioned cases of Extended Identity are adopted by, or have become strictly core identity factors. Joel Schumacher's Batman films $(1995,1997)$ are a good example and indication of the creator's interpretation and choices and of how these two adaptations and their Extended Identity manifestations may not strictly belong to the Core but pose more as a possibility of that Core. Extended Identities essentially offer multiple outlets for the abstract Core Identity to be realized. As far as adaptation theory is concerned, what has to be transferred from the initial work to any adaptation is the Core of the brand. A superhero is not a superhero unless a super-prefix exists in combination with a good versus evil battle and Manichean dichotomies of one form or another are present. How the Extended Identity is treated and, more importantly, to what degree it manifests the abstract elements of the Core Identity is what should concern adaptation theory. Any additional Extended Identity factors that simply act as details, or textures such as the aesthetics of the setting, the theme music, the outfits and props, the story conceived each time, the camera angles, editing and mise-en-scene, the narrator, or even the spectacle should not dictate if an adaptation is faithful. Essentially it becomes clear that it is not a matter of being faithful to the original, for even the original was influenced, or inspired, by something prior, hence the notion of intertextuality, but it is a matter of being loyal to the Core abstract elements that grant the existence of the superhero brand character in particular, and of any brand - whether product or world - in general. This train of thought ultimately sees all adaptations as works in their own right but as adaptations faithful to not necessarily each other but to the abstract concepts from whence they originated. After examining how the Core Identity has been realized for each superhero brand, these factors will help in determining the extent to which this realization was received, thus the Equity, or Value appointed to each brand. Hence, fidelity issues either pertaining to adaptation theory, or regarding the treatment of brands in general, ought to be re-assessed as a process whereby one examines what was done, how and what is its value and meaning in juxtaposition with the initial work as well as fellow works so as to determine what is considered more successful and of more value when dealing with a range of superhero brands. 


\section{Brand Equity and the Core Identity}

A brand not only needs to have a consistent Core Identity but as a whole it needs to have value, a matter that is examined from the scope of Brand Equity. According to Aacker, "brand equity is a set of assets (liabilities) linked to a brand's name and symbol that adds to (or subtracts from) the value provided by a product or service to a firm and/or that firm's customers. The major asset categories are: Brand name awareness, Brand loyalty, Perceived quality and Brand associations" (1996, 7-8). The asset of awareness is one that "refers to the strength of a brand's presence in the consumer's mind" $(1996,10)$. In the case of American mainstream superheroes existing since the 1930s, awareness, if not immediate recognition, is almost a given for the majority of the superhero character brands. If the audience is not aware of all of them, then at least the most renowned can be named such as Superman, Batman, and Spiderman. Awareness is measured according to the different ways consumers remember a brand "ranging from recognition, to recall, to 'top of mind,' to dominant” $(1996,10)$. Recognition is gained from past exposure not necessarily involving where the brand was encountered, why it is different, or even to which product class it belongs. Simply recognizing a brand, according to psychology, can produce positive feelings towards it $(1996,10)$. Recognition is made easier when a brand is associated with a symbol or logo, like the $\mathrm{S}$ of Superman, or the golden arches of McDonalds. This is the case of blockbusters in the area of film. Since their marketing campaigns target the widest possible audience, it is difficult to avoid seeing some advertisement of some kind before the film hits the theatres. As Aacker points out, "when consumers see a brand and remember that they have seen it before, they realize that the company is spending money to support the brand" $(1996,10)$. Brand recall, on the other hand, is said to happen when a product class is mentioned and the name of a brand pops into consumer's minds as an example, or means of reference, for the product class. For example, if the product class heroes, or superheroes, comes up then it is no surprise if Superman or Batman are recalled, thus standing as examples for that particular product class. Brand name dominance is the extreme part of the scale where consumers can only think of the name of a particular brand as standing for the entire product class, as has happened for brand names such as Zerox, Kleenex, and Kellogg's. The asset of perceived quality is one that essentially interests the Hollywood Entertainment Industry as far as Box Office revenues and ancillary markets are concerned and of course the consumers and whether they are getting their money's worth by purchasing said brand products. In order to 
reach any conclusions regarding this factor then statistical analyses are necessary both from the side of the industry as well as from the side of the consumers. Loyalty, a third asset, in the area of branding is mainly concerned with the loyalty of the consumers with the brand product. Thus, for the cases of Intellectual Properties, and their adaptations, the matter is left up to the actual consumers. If they are pleased with said brand adaptation, whether it is extremely faithful, or simply influenced by the initial work, they will display their degree of loyalty. Of course, this cannot act as the sole force for considering whether an adaptation is considered faithful, as adaptation theory has been advocating mainly due the fact that the majority of the audience is unaware of the procedures necessary for the production of a comic book, film, or video game and more importantly because audience members are subjective to what they read, see or play as are creators in their interpretations. Depending on their preferences, they may feel that an adaptation has been satisfactory such as Sam Raimi's Spiderman trilogy (2002, 2004, and 2007) and not feel discontent that various details such as the absence of character Gwen Stacey, or the contraption created by Peter Parker to control his web-wrist output has not been acknowledged, or employed, in the films. Others, however, may feel utter disappointment that these details were not included. Thus, when it comes to adaptations, fidelity and loyalty are also matters that concern the audience and apparently the end product can never fully satisfy everyone.

Consumers have, therefore, been divided into loyalty segments ranging from the "noncustomers (those who buy competitor brands or are not product class users), to the price switchers (those who are price sensitive), to the passively loyal (those who buy out of habit rather than reason), to the fence sitters (those who are indifferent between two or more brands) and finally to the committed" (Aacker 1996, 22). The notions of loyalty and fidelity not only for brands in general but also for adaptations, as this article is seeking to display, are matters that concern the industry and the audience. Essentially, the analysis and examination of adaptations results in comparisons between original and adaptations as well as the degrees of faithfulness. Perhaps it is more constructive and conclusive to examine what has remained similar and what has changed so as to decipher the implications of these changes, no matter how minute and, moreover, establish that especially when adapting a brand across media, it will automatically lead to changes and differences. By applying brand identity theory and considering the importance of Brand Equity nonetheless this article points to the Core as the factor that remains constant and stable throughout while the Extended Identity is 
what is negotiable and can act either as an asset ensuring Equity, or depending on the treatment and end-product even a liability, thus also taking into consideration the superhero brand adaptations that were not well received and did not do well at the Box Office. The final asset, that of brand associations, is driven by the identity of the brand and what it stands for in consumers' mind. For example, the brand of heroes has the range of including superheroes and when mentioned could cause a consumer to bring to mind a specific, or multiple superheroes. Moreover, the colours blue, red, and yellow as well as the capital S bring to mind the Superman brand, or the letter X can be associated with the X-Men brand. The notion of intertextuality fits very well with this brand equity asset especially if the brands under consideration are Intellectual Properties. This also means that aspects such as the aforementioned of the brand need not be the only elements capable of driving associations. For example, Robert Louis Stevenson's The Strange Case of Dr. Jekyll and Mr. Hyde (1886) could easily drive one to make associations with the Hulk character or vice versa. Having thus seen the components of Brand Equity, it becomes obvious how vital, and necessary, Equity is for the endurance of any brand. What is more, by substituting loyalty and value for fidelity, the latter immediately comes under re-assessment in regards adaptations, the Industry as well as consumer audiences.

\section{Conclusion}

The aim of this article was to propose a theoretical concept for examining adaptations that is based on the actual practices of Media Franchise culture where Intellectual Properties are treated as character or world brands. The core identity of a Brand enables one to determine the stable, continuous abstract agent that drives any particular character, or world brand. By extension, narrative and medium/a further assist by displaying how the Core is manifested in the extended identity. This in turn, allows adaptation theory to re-assess its holdings on fidelity issues because it becomes evident that the Extended Identity is the aspect that is negotiable and in need of change for adaptations to occur. Thus by holding the Core as the stable agent and seeing its treatment across narrative(s) and medium/a it becomes clear that fidelity is not a notion that should consider how faithful the adaptation was to the original but the degree of loyalty of the product towards the Core and what that entails. Juxtaposing the Extended Identity with the Core as well as including factors that pertain to Brand Equity further allow one to conceive of the vast network of choices that essentially lead to a 
dialogue regarding the what and how of any brand treatment which ultimately results in stability via change but also leaves room for negotiating the meanings offered by Extended Identity and how that affects the understanding of the Core. Whether a superhero brand is conceived in a comic book, film or game s/he is still a superhero, whether Spiderman sports a contraption for his web-wrist output or not he is still Spiderman, whether Tim Burton or Christopher Nolan direct the Batman film, it is still a Batman film. Hence, the collaboration of Adaptation theory and Brand Identity theory can lead to a re-assessment of fallacies and issues which can lead to, in the words of Alan Moore, the following conclusion, "everything is exactly the same, except for the fact that it's all totally different" (Will Brooker 2012, 42).

\section{References}

Aaker, David A. 1996. Building Strong Brands. London: Free Press Business.

Brooker, Will. 2012. Hunting the Dark Knight: Twenty-First Century Batman. London and New York: I. B. Tauris.

Cartmell, Deborah and Imelda Whelehan, eds. 1999. Adaptations: From Text to Screen, Screen to Text. London and New York: Routledge.

Elliott, Kamilla. 2003. Rethinking the Novel/Film Debate. Cambridge: Cambridge University Press.

Grainge, Paul. 2008. Brand Hollywood: Selling Entertainment in a Global Media Age. London and New York: Routledge.

Hutcheon, Linda. 2006. A Theory of Adaptation. New York: Routledge.

Johnson, Derek. 2009. Franchising Media Worlds: Content Networks and the Collaborative Production of Culture. Diss. U of Wisconsin-Madison.

Leitch, Thomas M. 2003. Twelve Fallacies in Contemporary Adaptation Theory. Criticism vol. 45 no. 2 (Spring): 149-171. http://muse.jhu.edu/journals/crt/ summary/v045/45.2leitch.html. Last accessed 24. 09. 2014.

McHale, Brian. 1996. Postmodernist Fiction. London: Routledge. 